DOI: $10.21105 /$ joss. 03611

\section{Software}

- Review 七

- Repository cat

- Archive ¿

Editor: Kevin M. Moerman ¿ Reviewers:

- Qalexlib

- @nkeim

Submitted: 06 May 2021

Published: 26 October 2021

\section{License}

Authors of papers retain copyright and release the work under a Creative Commons Attribution 4.0 International License (CC BY 4.0).

\title{
ParticleTracker: a gui based particle tracking software
}

\author{
Mike I. Smith*1 and James G. Downs ${ }^{1}$ \\ 1 School of Physics, University of Nottingham, UK, NG7 2RD
}

\section{Summary}

Tracking the motion of objects in a video is an important part of the analysis in a diverse range of subject disciplines (Meijering, 2012; Rahman, 2019; Rose, 2020). It enables one to automate the extraction of quantitative information about size, shape, motion etc. A number of tracking softwares exist (Bradski, 2000; D. B. Allan, 2020; J. Tineveza, 2016) to help with this process but the code can be an entry barrier. Even for researchers with the necessary skills, developing the code and optimising the parameters requires a significant investment for each new project. Open source tools that can simplify and expedite this process, whilst remaining flexible and easy to extend by the end user, would help to make particle tracking accessible to a broader range of researchers.

\section{Statement of need}

ParticleTracker is a completely gui based particle tracking software that implements and integrates a range of commonly needed tools to help users efficiently develop a wide range of different types of particle tracking projects. Though the underlying code is written in python it can be used as stand alone executables, enabling those with little or no coding ability to make use of these tools. It is also algorithm agnostic providing a uniform interface to 3 commonly used approaches to tracking. It therefore provides an open source solution for new users to use particle tracking as a part of their research. At the same time the python code base is designed to make extending the project extremely simple. Extension of each part of the code can be accomplished as simply as adding the project specific code to a preconfigured template and adding a single python dictionary to a parameter file.

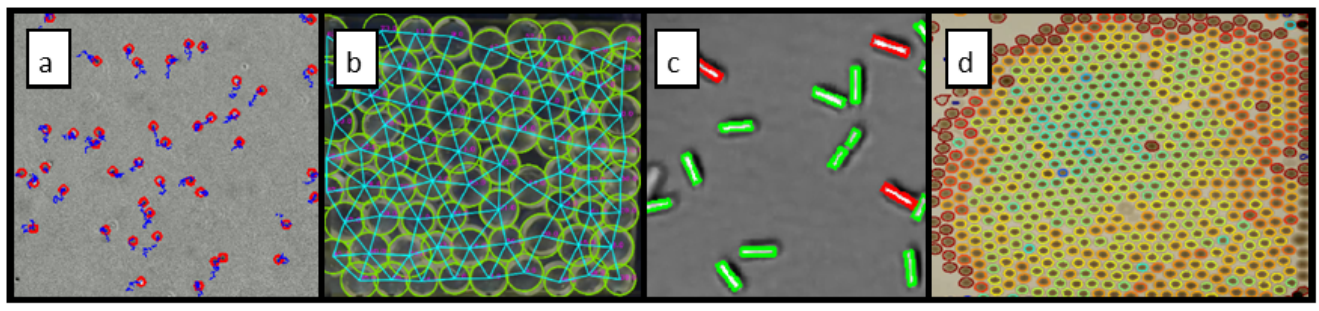

Figure 1: Example projects created using ParticleTracker (a) diffusing colloids (b) stress transmission in jammed birefringent discs (c) identifying dividing bacteria (d) swelling in hydrogel particles .

ParticleTracker was initially designed with the needs of masters students in mind who, in semester long projects, want to quickly move beyond writing code to track different types of objects (bubbles, bacteria, colloids, granular particles etc) and focus on the underlying science

*Corresponding author mike.i.smith@nottingham.ac.uk 
in their respective projects. Whilst the projects are varied, some of the underlying tools needed are often the same, with some small element that is specific to each project. The combined needs of efficiently setting up a new tracking project, an intuitive common interface for different underlying algorithms, but with the ability to easily extend some small part of it has therefore guided our design philosophy. Figure 1 illustrates a few example tracking projects (a) diffusing colloids (b) jamming in birerefringent discs (c) classifying dividing bacteria, (d) swelling hydrogels, which with a little practise can be setup in a few minutes. However, as the software has developed it has become clear that the development speed, uniform interface to different underlying methods, and versatility of the project would also be useful for larger scale research projects. Especially since it can be used by those with or without significant coding experience.

\section{Acknowledgements}

We acknowledge contributions from Nathan Smith in the early stages of this project. Mike Smith and James Downs acknowledge financial support from the Royal Society (UK).

\section{References}

Bradski, G. (2000). The OpenCV Library. Dr. Dobb's Journal of Software Tools.

D. B. Allan, N. C. K., T. Caswell. (2020). Trackpy: Fast, flexible particle-tracking toolkit. In GitHub repository. GitHub. https://doi.org/10.5281/zenodo.3492186

J. Tineveza, J. S., N. Perry. (2016). TrackMate: An open and extensible platform for singleparticle tracking. In Methods. Elsevier. https://doi.org/10.1016/j.ymeth.2016.09.016

Meijering, O. I., N. Dzyubachyk. (2012). Methods for Cell and Particle Tracking. Methods in Enzymology, 504. https://doi.org/10.1016/B978-0-12-391857-4.00009-4

Rahman, A. u. et al. (2019). Detection and tracking of bubbles in two phase air water flow for non convergent sinusoidal channel. IET Image Processing, 13. https://doi.org/10. 1049/iet-ipr.2018.5684

Rose, K. et al. (2020). Particle tracking of nanoparticles in soft matter. J. Applied Physics, 127. https://doi.org/10.1063/5.0003322 